\title{
Calculation of the interfacial free energy of a fluid at a static wall by Gibbs-Cahn integration
}

\author{
Brian B. Laird ${ }^{1, a)}$ and Ruslan L. Davidchack ${ }^{2}$ \\ ${ }^{1}$ Department of Chemistry, University of Kansas, Lawrence, Kansas 66045, USA \\ ${ }^{2}$ Department of Mathematics, University of Leicester, Leicester LE1 7RH, United Kingdom
}

(Received 20 February 2010; accepted 19 April 2010; published online 24 May 2010)

\begin{abstract}
The interface between a fluid and a static wall is a useful model for a chemically heterogeneous solid-liquid interface. In this work, we outline the calculation of the wall-fluid interfacial free energy $\left(\gamma_{\mathrm{wf}}\right)$ for such systems using molecular simulation combined with adsorption equations based on Cahn's extension of the surface thermodynamics of Gibbs. As an example, we integrate such an adsorption equation to obtain $\gamma_{\mathrm{wf}}$ as a function of pressure for a hard-sphere fluid at a hard wall. The results so obtained are shown to be in excellent agreement in both magnitude and precision with previous calculations of this quantity, but are obtained with significantly lower computational effort. (C) 2010 American Institute of Physics. [doi:10.1063/1.3428383]
\end{abstract}

\section{INTRODUCTION}

The solid-liquid interfacial free energy, $\gamma_{\mathrm{sl}}$, is a controlling parameter for a number of technologically important phenomena, including crystal growth and nucleation, dendritic morphology, and wetting. ${ }^{1-3}$ However, $\gamma_{\mathrm{sl}}$ is difficult to measure experimentally and accurate direct measurements only exist for a few materials. The dearth of experimental data has increased the importance of molecular modeling for solid-liquid interfaces, spurring the development of methods for deducing $\gamma_{\mathrm{sl}}$ from atomistic simulations, for example, cleaving methods ${ }^{4,5}$ and the capillary fluctuation method. ${ }^{6}$ Such methods have been used to determine $\gamma_{\mathrm{sl}}$ for a number of systems ranging from idealized model systems (for example, hard spheres, ${ }^{5,7,8}$ inverse power potentials, ${ }^{7}$ and Lennard-Jones potentials ${ }^{9-11}$ ) to systems modeling real materials (e.g., metals, ${ }^{6,12-15}$ metal alloys, ${ }^{16}$ and molecular materials $\left.^{17,18}\right)$.

The studies outlined above all involve the interface between a fluid and a fully dynamic and interacting solid phase. For chemically heterogeneous interfaces, however, a common approximation is to model the solid phase as a static surface or "wall" that functions as an external field confining the capillary fluctuations. For the calculation of the wall-fluid interfacial free energy, $\gamma_{\mathrm{wf}}$, for such systems either thermodynamic or mechanical techniques can be employed. Thermodynamic methods, such as thermodynamic integration using cleaving potentials ${ }^{19,20}$ and grand canonical transition matrix Monte Carlo, ${ }^{21}$ can be quite precise for wall-fluid systems; however, this precision comes at significant computational expense. Mechanical approaches rely on the equality of surface free energy and surface tension, which is true for a fluid-solid interface as long as the solid is treated as a static external field and not a fully interacting elastic system. ${ }^{22}$ In this method, ${ }^{23-26} \gamma_{\mathrm{wf}}$ is determined from simulation using the Kirkwood-Buff equation ${ }^{27}$ for the surface tension,

${ }^{a)}$ Electronic mail: blaird@ku.edu.

$$
\gamma_{\mathrm{wf}}=\int_{-\infty}^{\infty}\left[p_{n}(z)-p_{t}(z)\right] d z
$$

where $p_{n}$ and $p_{t}$ are the normal and tangential components of the pressure tensor and $z$ is the direction normal to the wall. Typically, because local pressure fluctuations in simulations are often large and Eq. (1) represents the difference between two pressure measurements (a numerical procedure that magnifies relative error), considerable computational effort ${ }^{28}$ is required to produce acceptable precision using Eq. (1).

In this work, we discuss the determination of interfacial free energies for a fluid in contact with a static wall through the integration of adsorption equations based on Cahn's reformulation ${ }^{29}$ of the thermodynamics of Gibbs. ${ }^{30}$ Such approaches were recently successfully applied by Frolov and Mishin $^{31,32}$ to solid-vapor and solid-liquid interfacial free energies of metals and metal alloys and by Laird et al. to the Lennard-Jones solid-liquid interface. ${ }^{33}$ As a demonstration, we apply this formalism to the calculation of the interfacial free energy of a hard-sphere fluid at a hard wall.

\section{METHOD: GIBBS-CAHN INTEGRATION FOR A FLUID AT A STATIC SURFACE}

The derivation of adsorption equations for fluids at a static surface follows from Cahn's generalization of the interfacial thermodynamics of Gibbs. ${ }^{29,32}$ For a system consisting of an $r$-component fluid at a static surface, the total Gibbs energy is given by

$$
G=E-T S+P V,
$$

where $P, T, E, S$, and $V$ are the pressure, temperature, internal energy, entropy, and volume, respectively. In the absence of a surface, the Gibbs energy would be equal to that of the bulk phase 


$$
G_{b}=\sum_{i=1}^{r} \mu_{i} N_{i}
$$

where $N_{i}$ and $\mu_{i}$ are the number and chemical potential of particles of type $i$, respectively. The interfacial free energy, $\gamma$, is given by the difference (per unit area) between the Gibbs energy of the system with the interface and that of the bulk,

$$
\gamma A=G-G_{b}=E-T S+P V-\sum_{i=1}^{r} \mu_{i} N_{i} .
$$

The differential of this quantity is

$$
\begin{aligned}
d(\gamma A)= & d E-T d S-S d T+P d V+V d P-\sum_{i=1}^{r} \mu_{i} d N_{i} \\
& -\sum_{i=1}^{r} N_{i} d \mu_{i} .
\end{aligned}
$$

The differential for the energy is given by

$$
d E=T d S-P d V+\sum_{i=1}^{r} \mu_{i} d N_{i} .
$$

Substituting Eq. (6) into Eq. (5) gives

$$
d(\gamma A)=-S d T+V d P-\sum_{i=1}^{r} N_{i} d \mu_{i} .
$$

For a fluid at a static surface, in addition to Eq. (7), we have the Gibbs-Duhem equation for the bulk fluid,

$$
0=-S^{f} d T+V^{f} d P-\sum_{i=1}^{r} N_{i}^{f} d \mu_{i}^{f},
$$

where the superscript $f$ denotes the fluid.

Equations (7) and (8) form a set of two simultaneous linear equations. Using Cramer's rule, Cahn showed that one of the differentials $d x$ (e.g., $d P$ ) can be eliminated to give

$$
A d \gamma=-[S / X] d T+[V / X] d P-\sum_{i=1}^{r}\left[N_{i} / X\right] d \mu_{i},
$$

where $X$ is the variable conjugate to the displacement $d x$ and the notation $[Y / X]$ is defined as

$$
[Y / X]=\frac{1}{X^{f}}\left|\begin{array}{cc}
Y & X \\
Y^{f} & X^{f}
\end{array}\right|,
$$

where, again, $f$ denotes bulk fluid and quantities without this designation refer to the full inhomogeneous system (fluid +interface). In writing Eq. (9), we have also used the fact that the area, $A$, of the static surface is constant so that $d(\gamma A)=A d \gamma$.

For a single component system $(r=1)$, a convenient choice is $X=N$. With this choice the $d \mu$ term in Eq. (9) is identically zero [because the determinant in Eq. (10) is zero if two columns are identical], giving

$$
A d \gamma=-[S / N] d T+[V / N] d P .
$$

If we define the interfacial excess entropy and volume (per unit area) for this choice of $X$ by

$$
\begin{gathered}
\eta_{N}=\frac{1}{A}[S / N]=\frac{1}{A}\left(S-S_{f} \frac{N}{N_{f}}\right), \\
v_{N}=\frac{1}{A}[V / N]=\frac{1}{A}\left(V-V_{f} \frac{N}{N_{f}}\right),
\end{gathered}
$$

then Eq. (11) becomes

$$
d \gamma=-\eta_{N} d T+v_{N} d P
$$

where subscript $N$ denotes that these excesses are calculated with the choice $X=N$.

The use of Eq. (14) requires knowledge of the excess interfacial entropy, $\eta_{N}$, which is not directly obtainable from simulation. For the current purposes, there are two ways to remedy this problem. One is to replace $\eta_{N}$ in Eq. (14) with other more readily available quantities using the general relation ${ }^{29,32}$

$$
\begin{aligned}
\gamma & =\left\{[E / X]-T[S / X]+p[V / X]-\sum_{i=1}^{r} \mu_{i}\left[N_{i} / X\right]\right\} / A \\
& =e_{X}-T \eta_{X}+p v_{X}-\sum_{i=1}^{r} \mu_{i} \Gamma_{i, X},
\end{aligned}
$$

where $e_{X}, \eta_{X}, v_{X}$, and $\Gamma_{i, X}$ are the excess interfacial energy, entropy, volume, and number of particles of type $i$ corresponding to the particular choice of $X$. For a single component system with $X=N$, Eq. (15) becomes

$$
\eta_{N}=\frac{e_{N}+p v_{N}-\gamma}{T}=\frac{h_{N}-\gamma}{T},
$$

where we have defined the excess interfacial enthalpy, $h_{N}$ $=e_{N}+p v_{N}$. This expression for $\eta_{N}$ contains the target quantity $\gamma$; however, if the integration of Eq. (14) is performed starting at a reference temperature and pressure for which $\gamma$ is known, then the initial value of $\eta_{N}$ can be calculated from Eq. (16). If one does not know the value of $\gamma$ at any $T$ and $P$, then this procedure is, of course, not useful.

A more practical approach to this problem was proposed by Baidakov et al. $^{34}$ for liquid-vapor interfaces and, in a more general context, by Frolov and Mishin. ${ }^{31,32}$ From Eq. (14), we have that $\eta_{N}=-[\partial \gamma / \partial T]_{P}$. Thus, Eq. (15) for a single component system with $X=N$ becomes

$$
\gamma+T\left(\frac{\partial \gamma}{\partial T}\right)_{P}=e_{N}+p v_{N}=h_{N} .
$$

The left hand side of Eq. (17) is equal to $-T^{2}[\partial(\gamma / T) / \partial T]_{P}$ so Eq. (17) becomes

$$
\left[\frac{\partial(\gamma / T)}{\partial T}\right]_{P}=-\frac{h_{N}}{T^{2}},
$$

from which Eq. (14) can be rewritten as 


$$
d(\gamma / T)=-\frac{h_{N}}{T^{2}} d T+\frac{v_{N}}{T} d P,
$$

which relates changes in $\gamma$ to the more readily obtainable excess interfacial enthalpy. The steps leading to Eq. (19) are analogous to the standard derivation of the Gibbs-Helmholtz equation in thermodynamics. ${ }^{35}$ Note that Eqs. (14) and (19) are not derivable from the standard Gibbs surface thermodynamics because the use of a Gibbs dividing surface in that formalism implies zero excess volume by definition. In this respect Cahn's extension of the Gibbs formalism is more general.

Another possible choice for a single component system is to set $X=V$, this gives

$$
d \gamma=-\eta_{V} d T+\Gamma_{V} d \mu .
$$

The Gibbs-Helmholtz form of this equation can be derived using the procedure described in the previous paragraph,

$$
d(\gamma / T)=-\frac{e_{V}-\mu \Gamma_{V}}{T^{2}} d T-\frac{\Gamma_{V}}{T} d \mu .
$$

Here $\eta_{V}, e_{V}$, and $\Gamma_{V}$ are the excess entropy, energy, and number of particles corresponding to the choice $X=V$.

For a multicomponent system, the choice of $X=V$ gives

$$
d \gamma=-\eta_{V} d T-\sum_{i=1}^{r} \Gamma_{i, V} d \mu_{i}
$$

where $\Gamma_{i, V}=\left[N_{i} / V\right]$ is the interfacial excess number of type $i$ particles for $X=V$ and $\mu_{i}$ is the chemical potential of particle type $i$. If there is no change in temperature $(d T=0)$, Eq. (22) becomes

$$
\left.d \gamma\right|_{T}=-\sum_{i=1}^{r} \Gamma_{i, V} d \mu_{i}
$$

which is the usual Gibbs adsorption isotherm. ${ }^{22}$ Alternatively, we can choose $X=N_{1}$, which gives

$$
d \gamma=-\eta_{N_{1}} d T+v_{N_{1}} d P-\sum_{i=2}^{r} \Gamma_{i, N_{1}} d \mu_{i} .
$$

As in the single component cases considered above, the differentials can be converted to the Gibbs-Helmholtz form to replace the dependence on the excess interfacial entropy with one on more readily obtainable interfacial excess quantities.

Each one of the adsorption differentials derived above can be integrated to give the change in the interfacial free energy, $\Delta \gamma$, along any chosen path in the space of the intensive variables $T, P$, and $\left\{N_{i}\right\}$. The determination of the value of $\gamma$ itself requires then that its value be known a priori at one reference point on this path, for example, through the use of one of the thermodynamic or mechanical integration techniques described above. Thus, these methods will be most useful when $\gamma$ for a specific system is needed for several points in thermodynamic space. The use of a computationally intensive direct method would only be required at one of the points, whereas the value of $\gamma$ at all other points could be obtained by integration of the Gibbs-Cahn adsorption equations, which is much less computationally intensive.
In Sec. III we examine the use of an adsorption equation in the calculation of $\gamma_{\mathrm{wf}}$ for a hard-sphere fluid at a hard, structureless wall.

\section{APPLICATION TO A HARD SPHERE FLUID AT A HARD WALL}

The best studied example of a fluid at a static surface is a single component hard-sphere fluid at a planar, structureless hard wall. For this system, the interparticle, $u(r)$, and wall-particle potential energies, $u_{\text {wall }}(z)$, are

$$
\begin{gathered}
u(r)= \begin{cases}\infty, & r<\sigma \\
0, & r \geq \sigma,\end{cases} \\
u_{\text {wall }}(z)= \begin{cases}\infty, & z<\sigma / 2 \\
0, & z \geq \sigma / 2,\end{cases}
\end{gathered}
$$

where $\sigma$ is the diameter of the particles, $r$ is the distance between two particle centers, and $z$ is the distance between a particle center and the wall position. In 1984, Henderson and van Swol used molecular-dynamics (MD) simulation to calculate $\gamma_{\mathrm{wf}}$ for this system mechanically using Eq. (1), although the precision of their results was quite low. Heni and Löwen $^{36}$ later improved upon the precision of results using the Monte Carlo simulation and thermodynamic integration with square barrier and triangular cleaving potentials to obtain $\gamma_{\mathrm{wf}}$ at several densities up to the solid-liquid coexistence density. More recently, Fortini and co-worker ${ }^{20,37}$ employed an exponential barrier function for the Monte Carlo thermodynamic integration to determine $\gamma_{\mathrm{wf}}$ at several pressures just below coexistence. Their results were consistent with those of Heni and Löwen, but were significantly more precise. Using an improved mechanical approach, De Miguel and Jackson ${ }^{28}$ obtained results for $\gamma_{\mathrm{wf}}$ for this system that although they were found to agree with the theoretical predictions of scaled particle theory (SPT) ${ }^{36,38,39}$ they were significantly smaller than those of Fortini et al. at densities near coexistence. The most recent calculations are those of Laird and Davidchack ${ }^{19}$ who used thermodynamic integration with a cleaving wall approach to calculate $\gamma_{\mathrm{wf}}$ over the full fluid range of densities. These results, which were reported to have a relative precision of about $0.1 \%$, were in full quantitative agreement with those of Fortini et al., even at the highest densities. In addition, the cleaving wall method allowed for the direct evaluation of $\gamma_{\mathrm{wf}}$ at the solid-fluid coexistence fluid density $\rho_{f}^{*}=\rho_{f} \sigma^{3}=0.9393$, whereas in the previous approaches this value could only be obtained by extrapolation from lower densities.

Both the mechanical and thermodynamic approaches to the calculation of $\gamma_{\mathrm{wf}}$ for the hard-sphere fluid/wall system require significant computational effort to achieve reasonable precision. In what follows, we use the Gibbs-Cahn integration formalism outlined above to determine high precision results $\gamma_{\mathrm{wf}}$ for this system with a significant reduction in computational effort.

For the hard-sphere fluid/hard-wall system considered here, we can assume constant temperature without loss of generality because the density profile is independent of $T$ and the interfacial free energy has a trivial linear scaling with $T$; 
TABLE I. Summary of results for excess volume $v$ and wall-fluid interfacial free energy $\gamma_{\mathrm{wf}}$ for the hard-sphere/hard-wall system as functions of density and pressure.

\begin{tabular}{lllll}
\hline \hline \multicolumn{1}{c}{$\rho^{*}=\rho \sigma^{3}$} & $P^{*}=P \sigma^{3} / k T$ & $v^{*}=v / \sigma$ & $v_{\mathrm{SPT}}^{*}=v_{\mathrm{SPT}} / \sigma$ & $\gamma_{\mathrm{wf}}^{*}=\gamma_{\mathrm{wf}} \sigma^{2} / k T$ \\
\hline 0.0 & 0.0 & 0.5 & 0.5 & 0.0 \\
$0.050715(2)$ & $0.056479(1)$ & $0.461(2)$ & 0.4620 & $0.02710(6)$ \\
$0.101326(3)$ & $0.125984(2)$ & $0.426(2)$ & 0.4281 & $0.05791(11)$ \\
$0.193195(6)$ & $0.295480(3)$ & $0.370(2)$ & 0.3738 & $0.1250(3)$ \\
$0.302903(11)$ & $0.600410(6)$ & $0.312(2)$ & 0.3196 & $0.2281(5)$ \\
$0.385260(13)$ & $0.935945(11)$ & $0.276(2)$ & 0.2848 & $0.3263(7)$ \\
$0.482523(17)$ & $1.506138(15)$ & $0.238(2)$ & 0.2490 & $0.4720(11)$ \\
$0.545621(17)$ & $2.01676(2)$ & $0.216(2)$ & 0.2283 & $0.5877(13)$ \\
$0.63666(2)$ & $3.03321(4)$ & $0.187(2)$ & 0.2014 & $0.792(2)$ \\
$0.69968(2)$ & $4.00290(4)$ & $0.1718(18)$ & 0.1846 & $0.965(2)$ \\
$0.749623(18)$ & $4.98307(7)$ & $0.1576(18)$ & 0.1722 & $1.126(3)$ \\
$0.79159(3)$ & $5.98980(10)$ & $0.148(2)$ & 0.1623 & $1.280(3)$ \\
$0.82850(3)$ & $7.04781(14)$ & $0.1378(17)$ & 0.1540 & $1.431(3)$ \\
$0.85743(2)$ & $8.01029(16)$ & $0.1303(16)$ & 0.1478 & $1.560(4)$ \\
$0.88434(2)$ & $9.03041(13)$ & $0.1231(17)$ & 0.1421 & $1.689(5)$ \\
$0.90721(2)$ & $10.00688(15)$ & $0.1153(16)$ & 0.1374 & $1.806(4)$ \\
$0.92712(2)$ & $10.9470(2)$ & $0.1099(13)$ & 0.1335 & $1.912(4)$ \\
$0.93900(2)$ & $11.5552(3)$ & $0.1044(13)$ & 0.1311 & $1.977(4)$ \\
\hline \hline & & & & \\
\hline
\end{tabular}

that is, $\gamma=\gamma^{*} k T / \sigma^{2}$, where $\gamma^{*}$ is dependent only on the density. For constant temperature, Eq. (19) becomes

$$
d \gamma=v_{N} d P
$$

Using Eq. (13), the excess volume per unit area, $v_{N}$, can be written in terms of the density profile, $\rho(z)$,

$$
v_{N}=\int_{0}^{\infty}\left[1-\frac{\rho(z)}{\rho_{f}}\right] d z
$$

where $\rho_{f}$ is the bulk fluid density. In the limit of zero density, where the density profile approaches a step function centered at $z=\sigma / 2$, the quantity $v_{N}$ approaches $1 / 2$.

We have performed MD simulations using the algorithm of Rapaport. ${ }^{40}$ The simulation box is tetragonal with hard walls placed at $z=0$ and $65 \sigma$. The $x-y$ cross section is square with a side length of $50 \sigma$. Periodic boundary conditions are employed in the $x$ and $y$ directions. The volume of the box is fixed and the bulk density and pressure are varied by changing number of particles. The number of particles ranges from about 8000 at the lowest pressure studied to about 150000 at the highest. The bulk densities and pressures are determined by averaging over the bulk region, which we define as the region farther than $12 \sigma$ away from either wall (that is, $12 \sigma$ $<z<53 \sigma$ ). We have determined that the effect of the walls in this region is significantly smaller than the statistical error in the bulk properties. The resulting bulk fluid densities, pressures, and excess volumes are summarized in Table I.

To find $\gamma$ as a function of $P$, we integrate Eq. (28) and use the fact that $\gamma$ will approach zero in the limit of zero pressure. To reduce the integration error, we subtract from $v$ the value of the excess volume per unit area according to SPT,

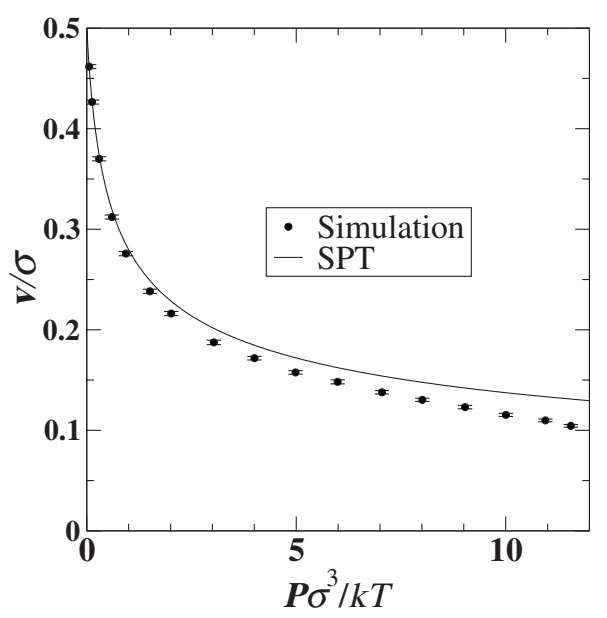

FIG. 1. Excess interfacial volume per unit area, $v$, for the hard-sphere/hardwall system as a function of reduced pressure. The black circles are the results of the current study. A 95\% confidence level is used to construct the error bars. The line shows this quantity as predicted by SPT using the Percus-Yevick expression for the pressure.

$$
v_{\mathrm{SPT}}=\frac{1-\eta}{2+4 \eta} \sigma
$$

where $\eta=\pi \rho \sigma^{3} / 6$ is the packing fraction. This expression was determined by differentiating the SPT expression ${ }^{38,39}$ for $\gamma$ with respect to $P$,

$$
\gamma_{\mathrm{SPT}}=P_{\mathrm{SPT}} \sigma / 2-\frac{9 \eta^{2}(1+\eta)}{2 \pi(1-\eta)^{3}} k T \sigma^{-2},
$$

where the SPT expression for pressure is

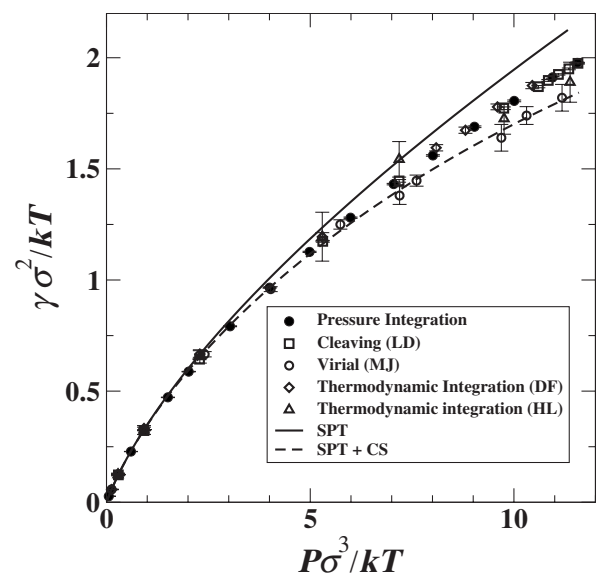

FIG. 2. Wall-fluid interfacial free energy per unit area, $\gamma_{\mathrm{wf}}$, for the hardsphere system as a function of reduced pressure. The black circles are the results of the current study. A 95\% confidence level is used to construct the error bars. For comparison, results from previous studies are also shown as open symbols: squares (Ref. 19, cleaving), circles (Ref. 28, virial), diamonds (Refs. 20 and 37, thermodynamic integration), and triangles (Ref. 36, thermodynamic integration). The lines show the predictions of SPT using the Percus-Yevick (solid line) or Carnahan-Starling (dashed line) expressions for the pressure. 


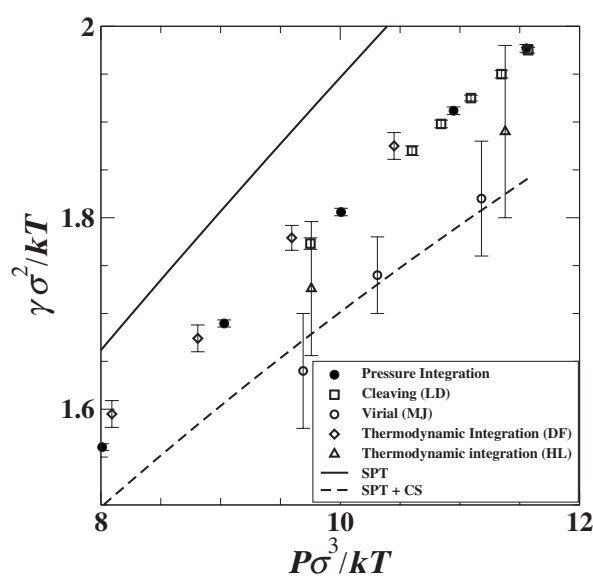

FIG. 3. Same as Fig. 2, except data are restricted to a reduced pressure range $\left[8<P \sigma^{3} / k T<12\right]$.

$$
P_{\mathrm{SPT}}=\frac{6 \eta\left(1+\eta+\eta^{2}\right)}{\pi(1-\eta)^{3}} k T \sigma^{-3}
$$

The calculated values of $v_{\mathrm{SPT}}$ at the pressures studied are also listed in Table I. A comparison of the excess volumes from simulation and SPT is shown in Fig. 1.

The values of $\gamma_{\mathrm{wf}}$ as a function of pressure are then determined by evaluation of the integral

$$
\gamma(P)=\gamma_{\mathrm{SPT}}+\int_{0}^{P}\left[v-v_{\mathrm{SPT}}\right] d P^{\prime}
$$

The integral is computed numerically using the trapezoid rule, which has an error for one step of $\left[-h^{3} f^{\prime \prime}(\xi) / 12\right]$, where $h$ is the step length, $f$ is the integrand, and $\xi$ is some point within the step interval. An upper bound of $f^{\prime \prime}$ within each step can be estimated from a polynomial fit of the integrand, from which we conclude that the integration error in the calculation of $\gamma_{\mathrm{wf}}$ is significantly smaller than the estimated statistical error. The calculated values of $\gamma_{\mathrm{wf}}$ are summarized in Table I. The computational effort to calculate $\gamma$ at the 17 different pressures from $P=0$ up to the coexistence pressure reported in Table I was comparable to that for the calculation of $\gamma$ at a single pressure using the cleaving method. ${ }^{19}$

Figure 2 shows the results of the current simulations with SPT and previous simulations. For better resolution of the high pressure results, Fig. 3 shows the same data as in Fig. 2 plotted over the pressure range of $8-12 k T / \sigma^{3}$. These figures show that the current results are in excellent agreement with the most precise previous simulation results, namely, the cleaving method calculations of Laird and Davidchack $^{19}$ and the thermodynamic integration calculations of Fortini and Dijkstra. ${ }^{20,37}$ The results also agree within the error bars with the thermodynamic simulation results of Heni and Löwen, ${ }^{36}$ but are at high pressures significantly larger than the results of the simulations of De Miguel and Jackson, ${ }^{28}$ who used a mechanical approach. In Figs. 2 and 3, two curves derived from SPT are shown. The upper (solid) curve uses Eq. (30) with the pressure evaluated using the standard SPT expression given in Eq. (31) and the lower (dashed) curve is the same, except that the pressure is evaluated using the Carnahan-Starling (CS) hard-sphere equation of state,

$$
P_{\mathrm{SPT}}^{\mathrm{CS}}=\frac{6 \eta\left(1+\eta+\eta^{2}-\eta^{3}\right)}{\pi(1-\eta)^{3}} k T \sigma^{-3} .
$$

\section{ACKNOWLEDGMENTS}

The computations were carried out on the University of Leicester Mathematical Modeling Centre cluster (purchased through the HEFCE Science Research Investment Fund). We also wish to thank Andrea Fortini for graciously providing us with data from his Ph.D. thesis. In addition we thank one of the referees for pointing out errors in our original derivations.

${ }^{1}$ D. P. Woodruff, The Solid-Liquid Interface (Cambridge University Press, London, 1973).

${ }^{2}$ J. M. Howe, Interfaces in Materials (Wiley, New York, 1997).

${ }^{3}$ A. W. Adamson and A. P. Gast, Physical Chemistry of Surfaces (WileyInterscience, New York, 1997).

${ }^{4}$ J. Q. Broughton and G. H. Gilmer, J. Chem. Phys. 84, 5759 (1986).

${ }^{5}$ R. L. Davidchack and B. B. Laird, Phys. Rev. Lett. 85, 4751 (2000).

${ }^{6}$ J. J. Hoyt, M. Asta, and A. Karma, Phys. Rev. Lett. 86, 5530 (2001).

${ }^{7}$ R. L. Davidchack and B. B. Laird, Phys. Rev. Lett. 94, 086102 (2005).

${ }^{8}$ M. Amini and B. B. Laird, Phys. Rev. B 78, 144112 (2008).

${ }^{9}$ R. L. Davidchack and B. B. Laird, J. Chem. Phys. 118, 7651 (2003).

${ }^{10}$ J. R. Morris and X. Song, J. Chem. Phys. 119, 3920 (2003).

${ }^{11}$ C.A. Becker, D. Olmsted, M. Asta, J.J. Hoyt, and S.M. Foiles, Phys. Rev. Lett. 98, 125701 (2007).

${ }^{12}$ J. J. Hoyt and M. Asta, Phys. Rev. B 65, 214106 (2002).

${ }^{13}$ D. Y.Sun, M. Asta, J. J. Hoyt, M. I. Medelev, and D. J. Srolovitz, Phys. Rev. B 69, 020102(R) (2004).

${ }^{14}$ H. M. Lawler, E. K. Chang, and E. L. Shirley, Phys. Rev. B 69, 174104 (2004).

${ }^{15}$ J. Morris, Phys. Rev. B 66, 144104 (2002).

${ }^{16}$ M. Asta, J.J. Hoyt, and A. Karma, Phys. Rev. B 66, 100101(R) (2002).

${ }^{17}$ X. Feng and B. B. Laird, J. Chem. Phys. 124, 044707 (2006).

${ }^{18}$ R. Handel, R. L. Davidchack, J. Anwar, and A. Brukhno, Phys. Rev. Lett. 100, 036104 (2008).

${ }^{19}$ B. B. Laird and R. L. Davidchack, J. Phys. Chem. C 111, 15952 (2007).

${ }^{20}$ A. Fortini and M. Dijkstra, J. Phys.: Condens. Matter 18, L371 (2006).

${ }^{21}$ E. M. Grzelak and J. R. Errington, J. Chem. Phys. 128, 014710 (2008).

${ }^{22}$ W. A. Tiller, The Science of Crystallization: Microscopic Interfacial Phenomena (Cambridge University Press, New York, 1991).

${ }^{23}$ G. Navascués, Rep. Prog. Phys. 42, 1131 (1979).

${ }^{24}$ J. R. Henderson and F. van Swol, Mol. Phys. 51, 991 (1984).

${ }^{25}$ M. J. P. Nijmeijer, C. Bruin, A. D. Bakker, and J. M. J. van Leeuwen, Phys. Rev. A 42, 6052 (1990).

${ }^{26}$ J. Z. Tang and J. G. Harris, J. Chem. Phys. 103, 8201 (1995)

${ }^{27}$ J. G. Kirkwood and F. P. Buff, J. Chem. Phys. 17, 338 (1949).

${ }^{28}$ E. De Miguel and G. Jackson, Mol. Phys. 104, 3717 (2006).

${ }^{29}$ J. W. Cahn, in Interfacial Segregation, edited by W. C. Johnson and J. M. Blakely (ASM International, International Materials Park, OH, 1979), pp. 3-23.

${ }^{30}$ J. W. Gibbs, The Collected Works (Yale University Press, New Haven, 1957), Vol. 1.

${ }^{31}$ T. Frolov and Y. Mishin, Phys. Rev. B 79, 045430 (2009).

${ }^{32}$ T. Frolov and Y. Mishin, J. Chem. Phys. 131, 054702 (2009).

${ }^{33}$ B. B. Laird, R. L. Davidchack, Y. Yang, and M. Asta, J. Chem. Phys. 131, 114110 (2009).

${ }^{34}$ V. G. Baidakov, S. P. Protsenko, and G. G. Chernykh, Russ. J. Phys. Chem. 80, 1519 (2006).

${ }^{35}$ R. A. Alberty and R. J. Silbey, Physical Chemistry, 3rd ed. (Wiley- 
Interscience, New York, 2000).

${ }^{36}$ M. Heni and H. Löwen, Phys. Rev. E 60, 7057 (1999).

${ }^{37}$ A. Fortini, Ph.D. thesis, Utrecht University, 2007.

${ }^{38}$ H. Reiss, H. L. Frisch, E. Helfand, and J. L. Lebowitz, J. Chem. Phys.
32, 119 (1960)

${ }^{39}$ J. A. Barker and D. Henderson, Rev. Mod. Phys. 48, 587 (1976).

${ }^{40}$ D. C. Rapaport, The Art of Molecular Dynamics Simulation, 2nd ed. (Cambridge University Press, New York, 2004). 